\title{
Effects of simulated predator eye illumination on tonic-immobility duration in Coturnix quail
}

\author{
JOHN B. CARMAN \\ Kansas Newman College, Wichita, Kansas
}

\begin{abstract}
Gallup, Nash and Ellison (1971) reported that the presence of artificial eyes increased both susceptibility to induction of tonic immobility (TI) and duration of the immobility reaction in domestic chicks. Further, they reported differential effects for both susceptibility and duration for color and type of eye stimulus. One interpretation of the differential effects suggests that the eye stimuli individually differed with respect to incidental light reflectance. The present study investigated the relationship between artificial eye illumination and duration of restraint-induced TI in habituated Japanese quail (Coturnix japonica). Results suggest that the differences reported by Gallup et al. may be due to the relative brightness of the eye stimuli.
\end{abstract}

Tonic immobility (TI) describes a fear-induced defense reaction that is exhibited by members of the higher invertebrate phyla and many vertebrate species, including amphibians, reptiles, fowl, and mammals. Ratner (1967) suggested that TI represents the terminal reaction in a sequence of adaptive behaviors that are initiated by stimuli arising from predator proximity. According to this "defensive distance" hypothesis, prey exhibit freezing in response to distant visual and auditory predator cues, escape behavior in response to more proximate visual cues, defensive aggression in response to proximate visual-auditory cues and initial tactile contact, and TI in response to prolonged tactile contact or physical restraint. The immobility response, overtly characterized by skeletal muscular immobility and hypertonicity, diminishes the provocative activity of prey and thus may attenuate further predaceous attack (Ratner, 1967; Sargent \& Eberhardt, 1975; Thompson, Foltin, Boylan, Sweet, Graves, \& Lowitz, 1981). Under typical laboratory conditions, TI is induced through hand restraint by the experimenter, and immobility duration may extend from seconds to hours.

In addition to the effects of a wide variety of subject variables, susceptibility to TI induction and TI duration are subject to modification by several situational variables, including familiarity with the test situation and/or experimenter, force and duration of physical restraint, body position during restraint, and social condition of rearing. In general, variables that augment fear level increase susceptibility to $\mathrm{TI}$ induction and extend TI duration (Gallup, 1974).

One variable of prepotence in the extension of TI duration is that of eye contact between subject and

Reprints may be obtained from John B. Carman, Department of Psychology, Kansas Newman College, 3100 McCormick, Wichita, Kansas 67213. predator, simulated predator, or experimenter. Gallup, Nash, Donegan, and McClure (1971) demonstrated that eye contact provided by a stuffed Coopers hawk resulted in a three-fold increase in duration of restraintinduced TI in the domestic chick. Subsequently, Gallup, Cummings, and Nash (1972) reported that eye contact maintained by a human observer markedly increased duration of TI in domestic chicks, and Gallup (1972) reported a similar effect when eye contact was provided by exposure to mirror-image stimulation. Recently, Gallup and Suarez (1980) proposed a model of openfield behavior which suggests that experimenter eye contact with the subject mimics a predaceous encounter. Open-field behavior is interpreted as following a course of compromise between attempts to reinstate conspecific contact and predaceous evasion. Substantial supportive evidence for this view has been provided by Suarez and Gallup (1980, 1981a, 1981b, 1982).

To evaluate further the relation between eye contact and TI, Gallup, Nash, and Ellison (1971) exposed immobile domestic chicks to pairs of brown or yellow glass eyes. The eyes were mounted on the ends of wooden dowel rods that were suspended immediately above the chick's head. Two additional groups of immobile chicks were exposed to either bare wooden dowel rod ends or glass eyes that had been covered with black plastic tape. Mean response durations for immobility induced under the four stimulus sets were: dowel rod ends, $45.3 \mathrm{sec}$; taped eyes, $72.5 \mathrm{sec}$; brown eyes, $302.3 \mathrm{sec}$; and yellow eyes, $368.1 \mathrm{sec}$. During the first trial of exposure to the stimulus sets, all chicks confronted with the glass eyes were susceptible to immobility induction. Chicks exposed to taped eyes exhibited $73.3 \%$ susceptibility, whereas those confronted with bare dowel rod ends showed 53.3\% susceptibility.

Although Gallup, Nash, and Ellison (1971) did not offer explanations for the differential effects of the 
brown versus the yellow eyes or the taped versus the dowel rod ends, they do conclude that eye presence extends the duration of restraint-induced immobility and that susceptibility to TI is facilitated by eye contact.

The Gallup, Nash, and Ellison (1971) data suggested that the differential effects of both the two eye colors and the noneye stimuli could be due to the relative luminance of the stimulus pairs. Ambient illumination in the experimental setting (indirectly provided by a $100-\mathrm{W}$ bulb) might be expected to be differentially reflected by the yellow or brown glass eyes and black plastic tape or wooden dowel rod ends. Specifically, the yellow eyes should be more luminous than the brown eyes and thus appear to be brighter. Black plastic tape, due to its surface texture, should have greater reflectance than the rough dowel rod ends. Interpreting the data accordingly, a direct relationship exists between stimulus luminance and both susceptibility to immobility induction and duration of the immobility reaction.

The present study attempted to determine the relationship between illumination of artificial predator eyes and duration of restraint-induced TI in the Japanese quail.

\section{METHOD}

\section{Subjects}

Thirty-two 6-week-old male Coturnix quail (Coturnix japonica) served as subjects. The quail were hatched from eggs obtained from a commerical supplier and were maintained on commercial chick grower. Under a 12-h light photoperiod, the chicks were socially reared in three cages containing 12 birds each. Human contact was kept at a minimum.

\begin{abstract}
Apparatus
A flat-black triangular cardboard box $(58 \times 50 \times 35 \mathrm{~cm})$ $33 \mathrm{~cm}$ deep was used for the induction of TI. A $16 \times 10 \mathrm{~cm}$ opening on the wide side permitted entry of the experimenter's hands into the box. Only the minimum indirect light required to permit observation of the quail in the induction box was used. Room-ambient illumination was approximately $3.77 \mathrm{~lx}$. The artificial eye apparatus consisted of an aluminum-foillined steel can $13 \mathrm{~cm}$ long and $12.5 \mathrm{~cm}$ in diameter. Mounted through the diagonal of the screw-top lid were two rigid foillined paper tubes. The tubes extended $19 \mathrm{~cm}$ from the can lid, were $2 \mathrm{~cm}$ in diameter, and were $4 \mathrm{~cm}$ apart at their distant ends. Glued to the end of each tube was a 2-cm-diam yellow taxidermic glass eye with a 5-mm black pupil. Inside the steel can was a $60-\mathrm{W} 120-\mathrm{V}$ ac bulb that could be turned off or adjusted by a switch and variable resistor. The entire apparatus, excluding eyes, was painted flat black. When mounted on the induction box, the eye tubes angled down at 45 deg toward the subjectrestraint area, with the eyes approximately $9 \mathrm{~cm}$ above the head of the restrained subject. Digital millisecond clocks were used to time all events.
\end{abstract}

\section{Procedure}

Each subject was identified with color-coded leg bands. Throughout all procedures, restraint duration was $10 \mathrm{sec}$, intertrial interval was $3 \mathrm{~min}$, and immobility duration was timed from the release of the subject until the recovery criterion of upright posture had been achieved. A trial consisted of manually restraining the subject on its right side in the induction box and recording the time elapsed until the recovery criterion had been met. If the subject failed to remain immobile for at least $5 \mathrm{sec}$, a maximum of three additional attempts was made to induce
TI. If TI could not be induced, a 0 -sec score was recorded for that trial. Experimenter eye contact with the restrained subject was avoided at all times.

Initial habituation training. On each of three consecutive days, each subject was administered three habituation trials in the induction box. The subject was kept between trials in a $28 \times 28 \times 28 \mathrm{~cm}$ black box. Following habituation training, the subjects were assigned to one of four groups $(N=8)$ that were equated for means and variances based upon immobilityduration scores. The groups were then assigned randomly to treatment conditions, and subsequent testing occurred on following successive days.

Equated habituation training. Three additional habituation trials were administered to the subjects in each of the four equated groups. During these trials, the eye apparatus was attached to the induction box. The eyes were painted flat black, and no voltage was applied to the bulb inside the eye apparatus.

Treatment training. The subjects in each group were administered three immobility trials according to the preassigned treatment condition. Group assignments to treatments were: Group 1-unpainted eyes, high illumination $\left(\mathrm{I}_{2}=376.7 \mathrm{~lx}\right)$; Group 2-painted eyes, no illumination; Group 3-unpainted eyes, low illumination $\left(I_{1}=37.7 \mathrm{~lx}\right)$; and Group 4-unpainted eyes, no illumination $\left(I_{0}\right)$. Illumination levels of the eyes were measured at the eye surface and pupil center.

\section{RESULTS}

Difference scores for each subject $\mathrm{x}$ group were derived between the immobility-duration scores for the three trials of equated habituation and the three trials

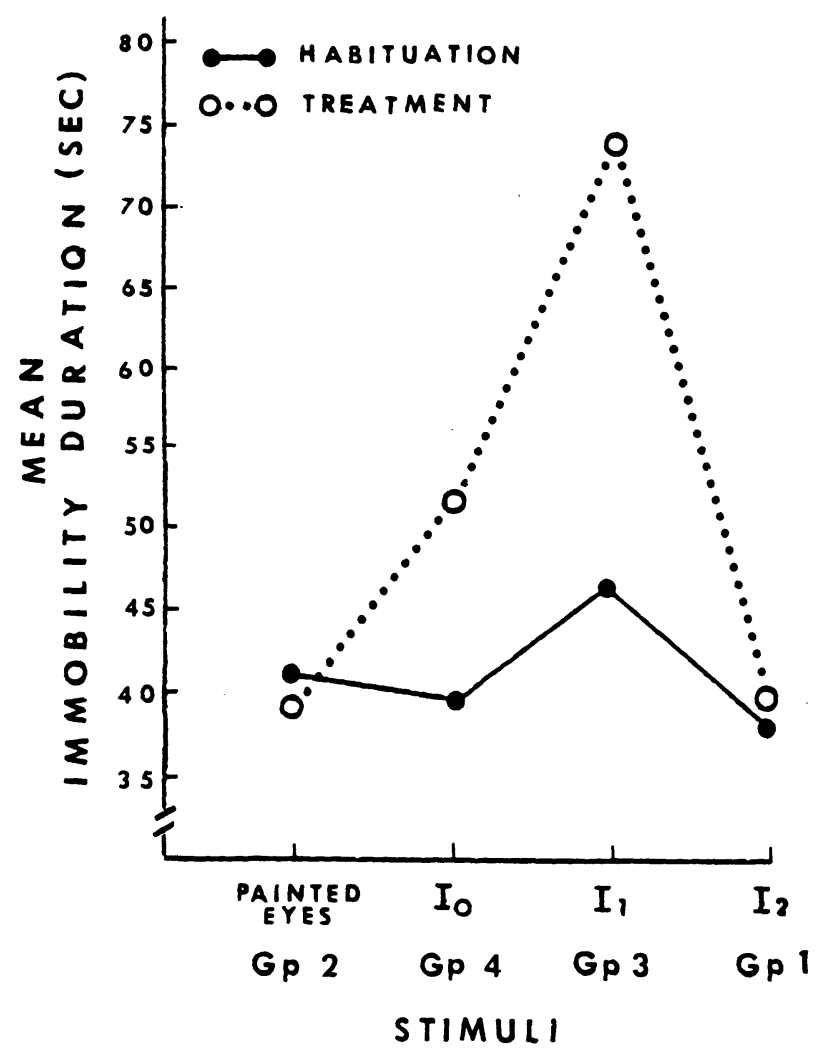

Figure 1. Mean immobility-duration scores for equated habituation and treatment training for the four treatment groups. 
of treatment training. Figure 1 depicts mean immobilityduration scores for equated habituation and treatment training for the four treatment groups. Analysis of variance of the difference scores revealed a significant treatments effect $[F(3,28)=3.03, p<.05]$. Using the difference scores for Group 2 as the reference, Dunnett's test indicated significant differences $(p<.01)$ for Groups 3 and 4. Directional within-group comparisons between means for equated habituation and treatment reached significance for Group 3 [ $t(7)=2.60, p<.025$ ], although the Group 4 difference was not reliable $(p<.1)$.

\section{DISCUSSION}

The results of the present study extend the potentiation of TI by simulated predator eye contact to another avian species and further support the reanalysis of the Gallup, Nash, and Ellison (1971) data relative to TI enhancement by the brightness of the eye test stimuli. The limit of TI enhancement, however, is reached at an extreme level of eye illumination. Enhancement of TI for Group 1 birds could have been masked by the relatively high illumination level in the induction box produced by the $I_{2}$ intensity, since Coturnix quail, unlike domestic chicks, exhibit substantially shorter TI duration at high ambient-illumination levels. An alternative view suggests that the $I_{2}$ illumination level may have eliminated iris-pupil contrast. This interpretation is supported by the finding of Gagliardi, Gallup, and Boren (1976) that the potentiation of TI by simulated predator eye contact is highly specific to the size of the pupil-to-eye diameter ratio. Of seven simulated eye pairs tested by Gagliardi et al., that pair with a pupil-to-eye ratio of $11 / 20 \mathrm{~mm}$ was found to be uniquely effective. As these investigators note, "what constitutes an 'eye' for a chicken may be remarkably specific" (p. 59). Thus, extreme brightness might obliterate the "eye" and perceptually reduce it to a simple source of illumination.

The defensive-distance hypothesis proposed by Ratner (1967) continues to be the most productive framework from which to view the behavioral consequences of TI. Eye contact between predator and prey, extensively investigated by Gallup and associates, adds an important dimension of stimulus specificity to the Ratner model. The TI-enhancing effect of eye illumination, which appears to parallel the "eye shine" luminosity produced by the tapetum lucidum of some predators, may further extend the model to crepuscular and nocturnal prey-predator interactions.

\section{REFERENCES}

Gagliardi, G. J., Gallup, G. G., Jr., \& Boren, J. L. (1976). Effect of different pupil to eye size ratios on tonic immobility in chickens. Bulletin of the Psychonomic Society, 8, 58-60.

GALLUP, G. G., JR. (1974). Animal hypnosis: Factual status of a fictional concept. Psychological Bulletin, 81, 836-853.

GalluP, G. G., JR. (1972). Mirror-image stimulation and tonic immobility in chickens. Psychonomic Science, 28, 257-259.

Gallup, G. G., Jr., Cummings, W. H., \& Nash, R. F. (1972). The experimenter as an independent variable in studies of animal hypnosis in chickens (Gallus gallus). Animal Behaviour, 20, 166-169.

Gallup, G. G., JR., Nash, R. F., Donegan, N. H., \& McClure, M. K. (1971). The immobility response: A predator-induced reaction in chickens. Psychological Record, 21, 513-519.

Gallup, G. G., JR., NAsh, R. F., \& Ellison, A. L., JR. (1971). Tonic immobility as a reaction to predation: Artificial eyes as a fear stimulus for chickens. Psychanomic Science, 23, 79-80.

Gallup, G. G., JR., \& Sunrez, S. D. (1980). An ethological analysis of open field behaviour in chickens. Animal Behaviour, 28, 368-378.

RATNER, S. C. (1967). Comparative aspects of hypnosis. In J. E. Gordon (Ed.), Handbook of clinical and experimental hypnosis. New York: Macmillan.

Sargent, A. B., \& Eberhardt, L. E. (1975). Death feigning by ducks in response to predation by red foxes (Vulpes fulva). American Midland Naturalist, 94, 108-119.

Suarez, S. D., \& Gallup, G. G., JR. (1980). An ethological analysis of open-field behavior in ducks (Ans platyrhynchos). Bird Behavior, 2, 93-105.

Suarez, S. D., \& Gallup, G. G., JR. (1981a). An ethological analysis of open-field behavior in rats and mice. Learning and Motivation, 12, 342-363.

Sunrez, S. D., \& Gallup, G. G., Jr. (1981b). Predatory overtones of open-field testing in chicks. Animal Learning \& Behavior, 9, 153-163.

Suarez, S. D., \& Gallup, G. G., JR. (1982). Open-field behavior in chickens: The experimenter is a predator. Journal of Comparative and Physiological Psychology, 96, 432-439.

Thompson, R. K. R., Foltin, R. W., Boylan, R. J., Sweet, A., Graves, C. A., \& Lowtiz, C. E. (1981). Tonic immobility in Japanese quail can reduce the probability of sustained attack by cats. Animal Learning \& Behavior, 9, 145-149.

(Manuscript received for publication November 5, 1983.) 